\title{
Correction: Whole-blood expression of inflammasome- and glucocorticoid-related mRNAs correctly separates treatment-resistant depressed patients from drug-free and responsive patients in the BIODEP study
}

Annamaria Cattaneo, Clarissa Ferrari, Lorinda Turner, Nicole Mariani, Daniela Enache, Caitlin Hastings, Melisa Kose, Giulia Lombardo, Anna P. McLaughlin, Maria A. Nettis, Naghmeh Nikkheslat, Luca Sforzini, Courtney Worrell, Zuzanna Zajkowska @, Nadia Cattane, Nicola Lopizzo, Monica Mazzelli, Linda Pointon (D, Philip J. Cowen, Jonathan Cavanagh, Neil A. Harrison, Peter de Boer, Declan Jones, Wayne C. Drevets, Valeria Mondelli, Edward T. Bullmore, the Neuroimmunology of Mood Disorders and Alzheimer's Disease (NIMA) Consortium, Carmine M. Pariante (iD

Correction to: Translational Psychiatry https://doi.org/10.1038/s41398-020-00874-7 published online 23 July 2020

We have corrected this Article post-publication, because Dr. Cattaneo's affiliation details were originally incorrect (she was affiliated with three institutions but is in fact only linked to one: Biological Psychiatry Unit, IRCCS Istituto Centro San Giovanni di Dio Fatebenefratelli, Brescia). These changes reflect in both the PDF and HTML versions of this Article.

Published online: 19 October 2020 